\title{
El financiamiento de las competencias profesionales del Banco Interamericano de Desarrollo (BID) en la educación superior tecnológica (IEST) de México
}

The financing of the professional skills of the Inter-American Development Bank (IDB) in technological higher education (IEST) of Mexico

\author{
Carlos Martínez Padilla * \\ Mireya Hernández Ramírez **
}

\begin{abstract}
Resumen:
Este artículo analiza la relación que tiene lugar entre el gobierno mexicano con organismos internacionales como el Banco Mundial (BM) y, el Banco Interamericano de Desarrollo (BID) para el financiamiento de las competencias profesionales en las Instituciones de Educación Superior Tecnológicas (IEST). Desde la perspectiva teórica de la Nueva Gestión Pública (NGP) se argumenta que el BID, a través de sus programas de competencias profesionales, tiene una gran influencia en las funciones $y$ atribuciones de las Universidades Politécnicas (UUPP) y, que dichos programas responden más a una lógica de compromisos institucionales que a una evaluación efectiva de resultados.
\end{abstract}

Palabras Clave: Educación Superior Tecnológica. Competencias profesionales. Banco Interamericano de Desarrollo (BID).

\begin{abstract}
:
This article analyzes the relationship, which is held between the Mexican government with international organizations like the World Bank (WB) and the Inter-American Development Bank (IDB) to finance professional competencies in Higher Education Technology Institutions (IEST). From the theoretical perspective of the New Public Management (NPM), we argue that the IDB, through its programs of professional competencies, has a great influence on the functions and attributions of the polytechnic universities (UUPP) and that such programs respond much more to a logic of institutional commitments than an effective assessment of results.
\end{abstract}

Keywords: University higher education. Professional competencies. Inter-American Development Bank (IDB).

\footnotetext{
* Doctor de Investigación en Ciencias Sociales. Profesor investigador de la Universidad Autónoma de Nuevo León (UANL). México. E mail: carlosmtzpadilla1@yahoo.com.mx

** Mtra. en Ciencias de la Educación. Profesora de la Universidad Icel. Distrito Federal, México. E mail: hrmireya2001@yahoo.com.mx
} 


\section{Introducción}

Las competencias profesionales en las Instituciones de Educación Superior Tecnológicas (IEST) tienen sus antecedentes en el Proyecto para la Modernización de la Educación Técnica y la Capacitación (Pmetyc), el cual estuvo conformado por cuatro componente ${ }^{1}$ que permitieron crear y consolidar un sistema de instituciones de capacitación, evaluación y certificación de competencias laborales en México a través del Consejo de Normalización y Certificación de Competencia Laboral (Conocer).

El Conocer fue creado en 1995, como un fideicomiso público no paraestatal entre la Secretaría del Trabajo y Previsión Social (STPS) y la Secretaría de Educación Pública (SEP). Sin embargo, para el 2003, el Gobierno Federal cuestionó su estatus jurídico por no operar como un órgano de gobierno ni como un Comité Técnico de un fideicomiso. Durante dos años, el Conocer abandonó sus actividades hasta obtener una nueva figura jurídica. En ese mismo año, el Banco Mundial evaluó negativamente el proyecto, con excepción del primer componente y, solo con algunas instituciones educativas del segundo componente, entre las que se encontraban las Universidades Tecnológicas (UUTT) ${ }^{2}$. Tales resultados, hicieron que el Banco Mundial, después de 35 años, dejara de financiar la educación técnica en México.

A pesar de la crisis institucional del Conocer, las competencias profesionales continuaron su avance y consolidación en las IEST. La Coordinación General de Universidades Tecnológicas (CGUT) dirigió el diseño y la elaboración de dos normas de competencia laboral desde 1999 en los distintos establecimientos del país: la norma de mantenimiento industrial y, la norma de gestión de la producción. Las normas fueron diseñadas con la metodología del Conocer y, elaboradas por profesores de las UUTT y, con personal directivo de industrias dinámicas y de prestigio del país.

Aunque la CGUT había convocado a las UUTT en 1999 a implementar las competencias profesionales en todos sus establecimientos de forma voluntaria, sólo

\footnotetext{
${ }^{1}$ Estos componentes son el Sistema de Certificación de Competencia Laboral (SCCL), la transformación de la oferta de formación y capacitación, los estímulos a la demanda de capacitación y certificación de competencia laboral y, los Sistemas de Información, Estudios, Evaluación y Administración del proyecto a cargo de la Unidad Administrativa del Pmetyc (UAPmetyc).

${ }^{2}$ Las Universidades Tecnológicas (UUTT) se incorporaron al Pmetyc en 1999 y, con ello, se marcó el inicio de las competencias profesionales en la Educación Superior Tecnológica en México.
} 
participaron los establecimientos de Jalisco, Tula, Hermosillo, Tabasco y Puebla. Posteriormente, llegaron a participar hasta 20 UUTT. No obstante, como el proyecto era voluntario, los directivos y administrativos de cada UUTT, no tenían la obligación de vigilar y seguir el Modelo de Educación Basado en Competencias Profesionales (MEBCP). Así, cuando terminaba un periodo de gobierno universitario, los nuevos directivos y administrativos de la universidad, no tomaban en cuenta el modelo, lo cual impedía que las competencias profesionales se llegaran a consolidar.

La institucionalización de las competencias profesionales en las IEST tuvo lugar a principios del 2002, cuando la Subsecretaria de Educación Superior e Investigación Científica $(\mathrm{SESIC})^{3}$ le solicitó a la CGUT la creación de un nuevo modelo educativo que distinguiera a las nacientes Universidades Politécnicas (UUPP) del resto de las IEST como los Institutos Tecnológicos Federales y los Tecnológicos descentralizados ${ }^{4}$.

Finalmente, los directivos y administrativos de la CGUT y de la SESIC decidieron crear el modelo educativo de las UUPP fundamentadas en el MEBCP. El modelo tenía como objetivo crear una universidad que respondiera a las necesidades de su entornó. Se buscaba que en las politécnicas tuvieran una gran vinculación, una gran dinámica con la sociedad y que sus programas de estudio constantemente se pudieran actualizar ${ }^{5}$.

A finales del 2003, se creó la Coordinación de Universidades Politécnicas (CUP) para diseñar e implementar el Modelo Educativo Basado en Competencias Profesionales (MEBCP) en los distintos establecimientos de las UUPP. En términos generales, el modelo tiene dos características singulares. Primero. Cuenta con órganos de gobierno, órganos de consulta y órganos unipersonales, en los cuales participan activamente diferentes actores del sector público, privado y social. Segundo. Todos sus planes y programas de estudio están diseñados al $100 \%$ basado en competencias profesionales ${ }^{6}$.

\section{La nueva gestión pública (NGP) y las IES}

\footnotetext{
${ }^{3}$.Hoy Subsecretaria de Educación Superior (SES).

${ }^{4}$.Hasta ese momento sólo habían iniciado actividades la primera universidad politécnica la Universidad Politécnica de San Luis Potosí en el 2001 y, la Universidad Politécnica de Tulancingo al año siguiente.

${ }^{5}$.Entrevista realizada al primer responsable del diseño e implementación del MEBC en las UUPP.

${ }^{6}$. Hasta el 2009 existían 34 Universidades Politécnicas (UUPP) que ofertan 33 programas académicos. Las UUPP están distribuidas en 21 estados de la República Mexicana, con una matrícula de un poco más de 26 mil estudiantes (http://politecnicas.sep.gob.mx).
} 
El Estado evaluador se consolidó en Europa cuando el Estado transformó sus políticas de evaluación a priori hacia una política de evaluación a posteriori (NEAVE, 1988). La evaluación a priori consistía en asignar recursos financieros a las Instituciones de Educación Superior (IES) de acuerdo a las metas y objetivos que se proponía alcanzar. Sin embargo, se observó que esta política resultaba poco efectiva porque el financiamiento se había entregado por "adelantado" sin saber si se iban a cumplir sus metas y objetivos planteados. Los hechos mostraban que muchas de las metas que se proponía alcanzar la IES no se cumplían. Por tanto, el Estado empezó a asignar recursos financieros en función del grado de cumplimiento de las metas y objetivos alcanzados por las IES. A mayor cumplimiento de metas y objetivos, las IES recibirían mayores recursos estatales.

A partir de esta nueva relación entre Estado e IES, se ha transitado de un centralismo estatal hacia una autorregulación o descentralización de la educación superior. Esta autorregulación o descentralización se expresó en la autonomía que otorgó el Estado a las IES. La autonomía consistía, por un lado, en que el Estado dejara de intervenir o controlar los procesos de educación superior en las IES como serían los planes de estudio, la orientación hacia determinadas disciplinas o, el acceso a la educación superior, para centrase en los productos finales tales como el número de graduados, patentes obtenidas, proyectos realizados o publicaciones realizadas. En ese sentido el Estado convierte a la educación superior en una "fábrica de conocimientos" (NEAVE; Van VUGHT, 1994).

Las políticas post evaluativas transformaron el papel que tenía el Estado en la asignación de recursos financieros a las IES. Si por mucho tiempo, la asignación se llevaba a través de los insumos que éstas solicitaban para realizar funciones de docencia e investigación, a finales de la década de los ochenta, el Estado empezó a asignar recursos en base a productos finales que habían realizado las IES.

El Estado evaluador se fortaleció con el arribo de la Nueva Gestión Pública (NGP) ${ }^{7}$ La NGP ha tenido una influencia significativa en los gobiernos de varias partes del mundo y, tiene como objetivo reemplazar las culturas administrativas, jerárquicas y profesionales que tenido los gobiernos por una cultura privada, comercial y de mercado. La NGP ha contribuido eficientar los ingresos públicos, mejorar la satisfacción de los ciudadanos como

\footnotetext{
${ }^{7}$ La NGP fue impulsada por organismos internacionales como la organización para la Cooperación y el Desarrollo Económico (OCDE) y el Banco Mundial (BM) durante la década de los noventa.
} 
receptores de los servicios que ofrece el gobierno, delegar responsabilidades a los niveles inferiores del gobierno y al sector privado, mejorar la capacidad para elaborar, implementar y medir las políticas públicas, entre otros aspectos (LYNN, 2004). La NGP intenta reproducir las formas de la gestión del sector privado en el sector público, es decir, hacer eficientes los ingresos públicos y mejora la satisfacción de los ciudadanos, quienes son los que reciben los servicios del gobierno.

En Europa la NGP contribuyó a mejorar la autonomía universitaria. Éstas se encargaban de cumplir las metas planteadas por el gobierno, utilizando sus propios criterios y medios que mejor consideran necesarios. Para el Estado, a mayor autonomía de las IES mejor desempeño podían tener con respecto a las metas establecidas por el gobierno (MAGALHAES; AMARAL, 2009).

En México, la crisis económica de los años ochenta obligó al gobierno mexicano a diseñar políticas financieras restrictivas que habían estado sustentadas en los insumos que requerían las universidades y, no en los productos finales que generaban. Los recursos eran asignados de acuerdo con los insumos requeridos por las universidades tales como el número de alumnos declarados por la IES ante la $\mathrm{SESIC}^{8}$; el número de profesores o administrativos contratados en la universidad, o bien, por las negociaciones políticas que tenían los rectores de las universidades con el gobierno federal. De igual forma, los salarios de estos actores eran determinados por la homologación salarial que hubiera conseguido la UNAM o, por los aumentos salariales establecidos en los pactos para la estabilidad económica (KENT; DIDOU; VRIES, 2001).

Posteriormente, en la década de los años noventa, se invirtió la relación de la asignación de recursos basada en los insumos que requerían las universidades, hacia una asignación de recursos basada en productos finales orientada hacia el fomento de la investigación o el mejoramiento al desempeño académico. Esta nueva forma de asignación de recursos financieros dio paso al establecimiento de un Estado Evaluador. A partir de entonces, las políticas de financiamiento público se han orientado a mejorar los niveles de calidad de los programas educativos y servicios a través de proyectos que realizan las mismas IES, en especial las Universidades Públicas Estatales (universidades autónomas de

\footnotetext{
${ }^{8}$ Subsecretaria de Educación Superior e Investigación Científica (SESIC), hoy Subsecretaria de Educación Superior (SES).
} 
los estados) - UPES - y las Universidades Públicas con Apoyo Solidarios (Universidades públicas descentralizadas) -UPEAS-. El cumplimiento de los objetivos de tales proyectos les permite a las IES obtener recursos extraordinarios ${ }^{9}$ otorgados por la Secretaria de Educación Pública (SEP).

Asimismo, la NGP permitió la participación de nuevos actores que median entre el Estado y la Universidad como son las agencias nacionales de evaluación. En ese sentido, se puede señalar que parte de la autoridad central que había tenido el Estado es cedida a tales agencias nacionales que evalúan la calidad de la educación superior. Las políticas de evaluación centradas en la calidad promueven la rendición de cuentas (accountability) a través de informes de productos finales realizados. La rendición de cuentas, ya sea de tipo legal, financiera, o de carácter académico propiciaron que las IES estuvieran obligadas a cumplir con modelos y requisitos de evaluación para obtener recursos extraordinarios del Estado (DEL CASTILLO, 2005).

La NGP criticó la forma colegiada de gobierno por considerarla tanto ineficiente como no efectiva porque no responden a las demandas sociales y económicas que requiere la sociedad y, que en su lugar predomina la ciencia universal y, la libertad académica como valor sagrado. Considera que el deber del gobierno consiste en el bienestar y protección de la comunidad científica así como ofrecer el servicio de educación superior gratuito financiado a través de los impuestos (BRAUN; MERRIEN, 1999). En Europa se ha señalado que a medida que avanza la autonomía en las IES, se hace necesario reemplazar la gobernanza colegiada por un gobierno universitario que responda a las necesidades económicas y sociales contemporáneas (MAGALHAES; AMARAL, 2009).

\section{El Banco Interamericano de Desarrollo (BID) y la Secretaria de Educación Pública (SEP)}

A diferencia del Banco Mundial que financia programas orientados a la educación básica, el BID financiaba proyectos relacionados con programas de educación superior, programas de formación profesional, programas de educación que proporcionen destrezas sociales y de trabajo a jóvenes y adultos que no han tenido acceso a la educación normal, programas destinados a mejorar la organización escolar, el currículo y técnicas de

\footnotetext{
${ }^{9}$ Ver Cordera y Pantoja (1995), Casanova (2000) y, Mendoza (2009).
} 
enseñanza aprendizaje a nivel primario y secundario, así como programas que mejoren la eficiencia y eficacia el financiamiento educativo.

Desde el año 2000, el financiamiento del BID para la educación superior ha aumentado sustancialmente. Ha financiado proyectos para ayudar a estudiantes universitarios de bajos recursos, evitar la deserción escolar, enfrentar epidemias en espacios escolares, o bien, financiar la implementación de las competencias profesionales en la educación superior a través del Proforhcom (BID, 2004, 2005). ${ }^{10}$

El Proforhcom es implementado por la Secretaría de Educación Pública (SEP) a través de dos instancias. La primera instancia es la Unidad Coordinadora y Administradora del Programa (UCAP), la cual tiene dos funciones: coordinar la programación y ofrecer apoyo a las actividades de los diferentes Ejecutores Técnicos y; gestionar administrativamente el programa. La segunda instancia es el Comité Directivo del Programa (Codipro) que tiene como principal función el direccionamiento y coordinación estratégica del programa (ver Esquema 1).

Los ejecutores técnicos comprenden varias instituciones de la educación media superior, así como a la Dirección General de Educación Superior Universitaria (DGESU antes DGES $^{11-)}$ la cual está a cargo de las actividades en las Universidades Politécnicas y Universidades Tecnológicas. Los lineamientos que le impone el BID a la CUP son los establecidos en el Proforhcom Fase I y Fase II (BID, 2005, 2010). Uno de ellos señala que la CUP tiene la función de coordinar todas las actividades para implementar el Modelo de Educación Basado en Competencias (MEBC) en las Universidades Politécnicas (UUPP).

\footnotetext{
${ }^{10}$ A lo largo de la década del 2000, el BID había financiado ocho proyectos relacionados con la educación. Tres de ellos están relacionados con la educación superior, dos con las comunidades rurales y, el resto están dirigidos a las empresas, a la administración pública y al manejo de crisis del sector educativo a causa de la epidemia del virus de la influenza H1N1 -gripe porcina- (www.iadb.org).

${ }^{11}$ Dirección General de Educación Superior.
} 
Esquema 1 - Estructura Del Proforhcom

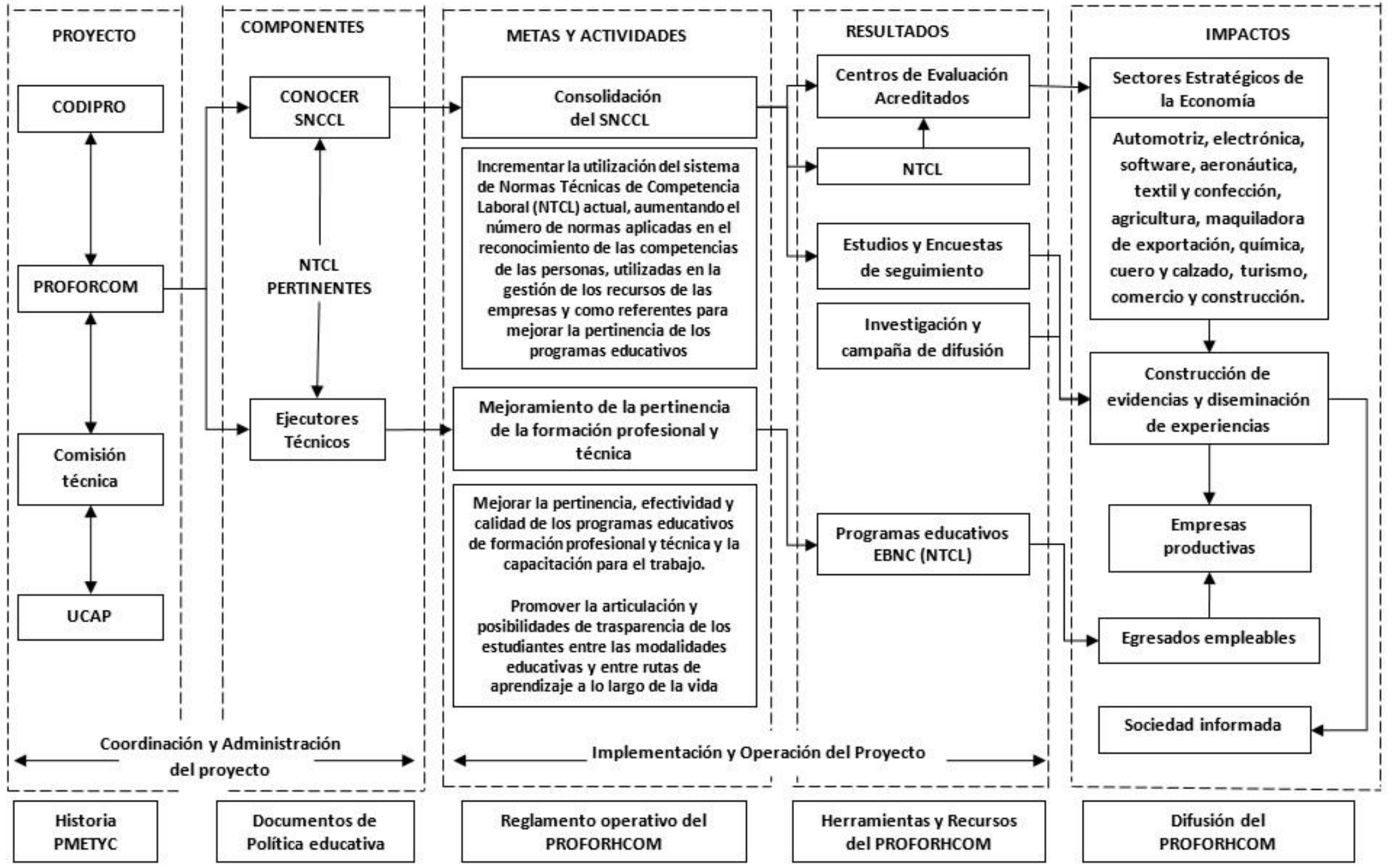

Fuente: UCAP (2007). 
Las atribuciones que tiene cada subsistema de educación superior tecnológico se encuentran en el reglamento interno de la Secretaria de Educación Pública (SEP), expedido en el 2005. Aunque el reglamento ha tenido tres reformas, no especifica las funciones de la Coordinación de Universidades Politécnicas (CUP).

Las atribuciones y funciones de la CUP se encuentran reguladas por el reglamento operativo del Proforhcom. El reglamento fue aprobado en el 2007 por Comité Directivo del Programa (Codipro) con el visto bueno del BID. El reglamento específica los objetivos de la CUP que consiste en incorporar el enfoque del MEBC en los programas de ingeniería existentes en las UUPP; promover y difundir el enfoque EBC entre los sectores productivos y las instituciones de educación superior; promover, entre los estudiantes y egresados del nivel medio superior, la formación en competencias a nivel superior; propiciar la continuación de estudios de los egresados formados en EBC en otras instituciones y; mejorar la empleabilidad de los egresados del nivel superior.

La gran mayoría del personal de la Coordinación de Universidades Politécnicas (CUP) son consultores y trabajan bajo el régimen de honorarios. La CUP ante el gobierno no es una unidad responsable, es decir, no es una unidad administrativa que pertenezca a una estructura básica de una dependencia ni tampoco está facultada para ejercer gasto con el fin de llevar a cabo actividades que conduzcan al cumplimiento de objetivos y metas propias. En ese sentido, la estructura organizacional y los puestos de trabajo en la CUP, legalmente no existen, porque la CUP no es una unidad responsable que dependa del Gobierno Federal. La estructura organizacional y los puestos de trabajo en la CUP son de forma simbólica y honorífica con el único objetivo de organizarse. Así podemos encontrar un departamento de planes y programas de estudio, finanzas, normatividad o espacios (Ver Esquema 2).

El pago a los consultores de la CUP es cubierto como parte del financiamiento del Proforhcom. Algunos puestos clave de la CUP, como la Coordinación de la CUP o la Responsable de Espacios, no son financiados por el Proforhcom, sino por la SEP, debido a que requiere de este respaldo jurídico para gestionar recursos, establecer acuerdos o convenios con otras dependencias gubernamentales. 
Esquema 2 - Organigrama de La CUP 2011

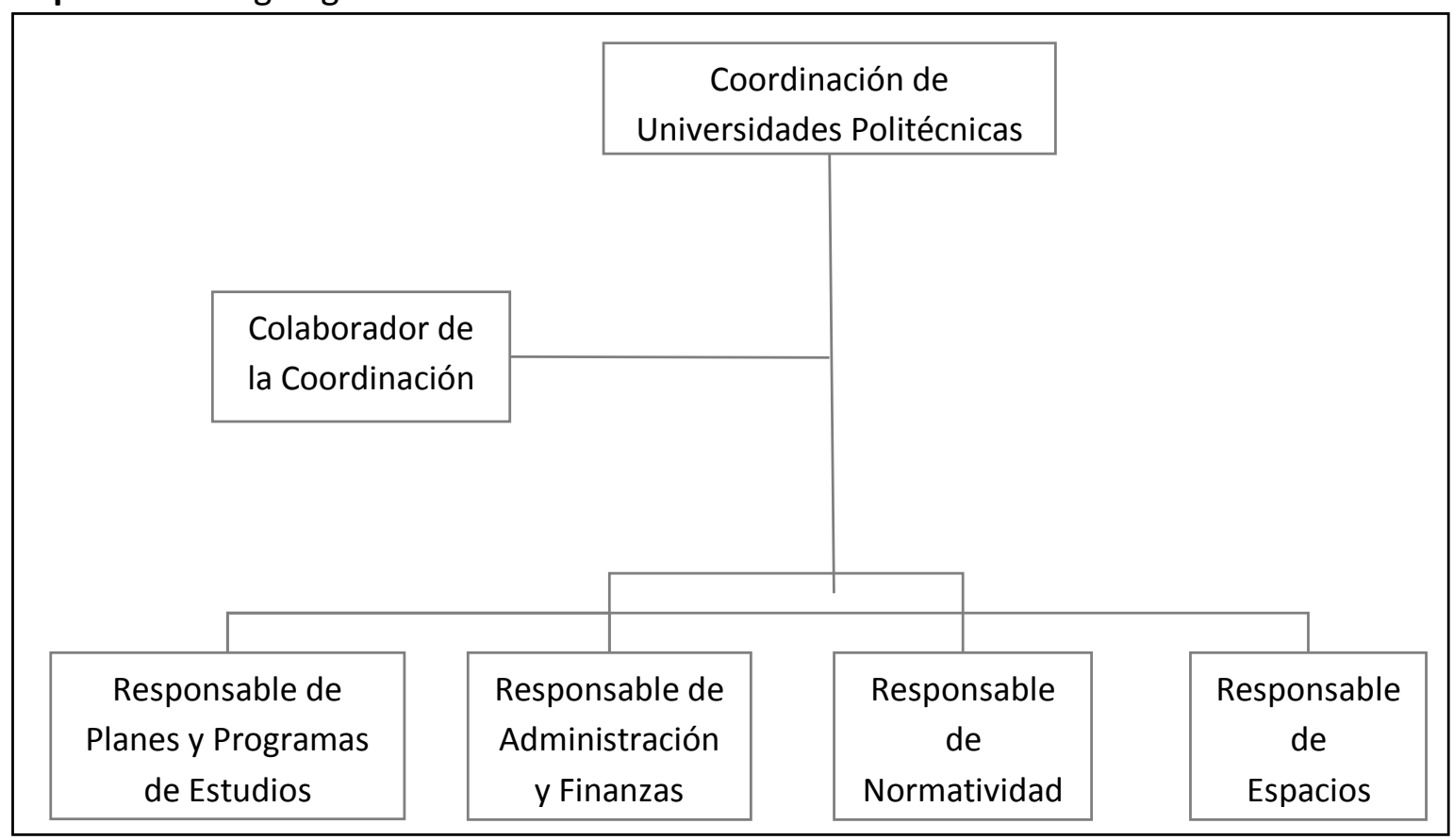

Fuente: Mexico (2011).

Todo el personal de la CUP, con las excepciones antes mencionadas, es contratado mediante el procedimiento de adjudicación directa, con fundamento en los artículos 26 fracción III y 42 de la Ley de Adquisiciones, Arrendamientos y Servicios del Sector Público. Tales artículos señalan que las dependencias y entidades gubernamentales deberán establecer en los procedimientos de contratación los mismos requisitos y condiciones para todos los participantes y proporcionar a todos los interesados igual acceso a la información relacionada con dichos procedimientos para evitar favorecer a algún participante.

En un principio, el Proforhcom Fase I, había señalado que las UUPP serían las encargadas de coordinar y dirigir a las Universidades Tecnológicas (UUTT) para implementar el MEBC en sus establecimientos. En el 2004, la Coordinación General de Universidades Tecnológicas (CGUT) y la Coordinación de Universidades Politécnicas (CUP), acordaron colaborar conjuntamente para que el MEBC fuera piloteado en las UUTT debido a que sus planes de estudio comprenden dos años mientras que los planes de estudio de las UUPP son de tres años y medio. Se tenía proyectado pilotear los efectos de la implementación del MEBC en las y, tomar las medidas necesarias en las segundas. Este objetivo se pudo apreciar en una de las primeras páginas electrónicas oficiales de la CUP del 2005, en la cual también se hace referencia al Proforhcom: 
La Misión de la Coordinación de Universidades Politécnicas (CUP) para la ejecución del proyecto está orientada a: Coordinar a las Universidades Tecnológicas y Politécnicas en el diseño, e implantación de los planes y programas de estudio bajo el modelo de competencias; asesorar y crear las condiciones para el análisis, la reflexión y la creatividad, que hagan posible que las propias instituciones elaboren sus currículos, trabajo de manera colegiada y en colaboración interinstitucional (MEXICO, 2007).

Las UUPP diseñaron la metodología para elaborar planes y programas de estudio en base a competencias e invitaron a varias UUTT a participar en el proyecto piloto. Trece universidades decidieron participar, superando así, lo establecido por el Programa de Formación de Recursos Humanos Basada en Competencias (Proforhcom) Fase I que requería como mínimo cinco UUTT.

\section{El Proforhcom Fase I y Fase II}

A finales de Septiembre del 2004 se había expedido la propuesta de préstamo ante el BID, con la cual se dio a conocer el Proforhcom Fase I. Allí se presentaron datos de las cuatro UUPP ${ }^{12}$ que hasta el momento existían. Los datos presentaban una significativa reducción de deserción en el primero año de estudios, el diseño e implementación de siete carreras de ingeniería en los planes y programas de estudios en base a Normas Técnicas de Competencia Laboral (NTCL) y, la capacitación de 90 profesores para "la instalación de las carreras EBC".

Asimismo, el Marco Lógico de la propuesta de préstamo del Proforhcom Fase I presentaba una condición para que pudiera operar el Proforhcom Fase II. La condición sostenía que el Subsistema de Universidades Politécnicas (SUP) debía de haber "desarrollado e incorporado 7 carreras en EBC en las Universidades Politécnicas existentes (BID, 2004)". Un año después, en abril del 2005, esta meta de desarrollo e incorporación de las carreras a EBC se cambio en el contrato de préstamo con el BID hacia una afinación de una metodología para el diseño de planes y programas de estudio en educación basada en competencias (BID, 2005).

\footnotetext{
12 Para el 2004 había cinco UUPP que habían iniciado actividades. El BID sólo contempló las primeras cuatro: la Universidad Politécnica de San Luis Potosí, la Universidad Politécnica de Tulancingo, la Universidad Politécnica de Aguascalientes y, la Universidad Politécnica de Zacatecas. La quinta politécnica fue la Universidad Politécnica de Puebla.
} 
El Proforhcom fase I, se dio a conocer en el 2005. Muchos de los lineamientos que establecía, habían sido ya cumplidos, incluso desde un año antes. Las siete carreras que señala el Proforhcom para implementar la Educación Basada en Competencias (EBC), existían desde la creación de las Universidades Politécnicas, al igual que los programas educativos de las UUTT que eran una continuación del Pmetyc. El Proforhcom Fase I, también se volvía parte de las políticas post evaluativas. EI BID a través del Gobierno Federal, sólo estaba financiando proyectos que previamente habían mostrado resultados (BID, 2005).

2.13 UT y UP. Dentro de su proceso de ampliación de la educación basada en competencias, las UT y UP afinarán una metodología para el diseño de planes y programas de estudio en educación basada en competencias que aplicará en siete (7) carreras de ingeniería (industrial, electrónica y telecomunicaciones, mecatrónica, biotecnología, telemática, tecnologías de la información y sistemas) de las cuatro (4) UP existentes, y en los programas de Mantenimiento Industrial y Procesos de Producción de técnico superior universitario en casos piloto de cinco (5) UT (BID, 2005, p. 17).

El Proforhcom Fase II profundiza la EBC y propone la capacitación de profesores y la participación de los representantes del sector productivo. El BID reconoció a través de la propuesta de préstamo del Proforhcom Fase II, en Diciembre del 2008, que las metas establecidas para las UUPP se habían cumplido ${ }^{13}$. Como hemos mencionado, las metas se habían cumplido porque el BID tomó como parámetro los avances que hasta ese momento habían realizado las UUPP. En dicha propuesta de préstamo el Proforhcom Fase II apoyaba

[...] cuatro aspectos clave para la transformación hacia un sistema basado en competencias: la articulación curricular como paso para la portabilidad de competencias; el desarrollo de competencias genéricas, disciplinarias y profesionales; la formación docente basada en competencias; y la participación de los sectores productivos en el proceso de definición de las competencias (BID, 2008, p. 6).

En general los primeros intentos de gestión del MEBC se encontraban a nivel de propuesta pero difícilmente se llevaron a cabo. Los primeros intentos llevados a la

\footnotetext{
${ }^{13}$ Aunque todavía no había concluido el Proforhcom Fase I, en diciembre del 2008 se dio a conocer la propuesta de préstamo para el Proforhcom Fase II. El Proforhcom Fase I, terminó en abril del 2010 y en febrero del 2010 se estableció el contrato para iniciar el Proforhcom Fase II (BID, 2009, 2010).
} 
realidad iniciaron con el pilotaje del MEBC en las UUTT y con el Proforhcom Fase I y II que financiaba el MEBC de acuerdo a los resultados "ya obtenidos por las UUPP".

\section{Conclusiones}

Tanto la política de evaluación a posteriori como la NGP transformaron considerablemente las funciones de las IES en México. La primera estableció nuevos objetivos a las IES como sería solicitar recursos financieros al Estado en función de los productos finales que habrían realizado. La segunda, transformó los procedimientos administrativos para incrementar los ingresos públicos y mejorar la satisfacción de los diferentes actores de las IES: estudiantes, académicos, personal administrativo y directivo.

Si en Europa la NGP llegó a mejorar la autonomía universitaria haciendo que cada IES utilizara sus propios criterios y medios para cumplir las metas planteadas por el gobierno, en México la autonomía universitaria se debilitó. La NGP promovió y estableció agencias nacionales de evaluación que le permitieron al Estado no sólo ser evaluador, sino también interventor en las IES. Estas agencias evalúan diferentes procesos de calidad en las IES que van desde la infraestructura hasta procesos de enseñanza aprendizaje.

Las UUPP se diferencian de otras IEST del país, no sólo con su MEBCP sino también en su forma de gobierno. La autoridad del gobierno universitario de las Universidades Tecnológicas (UUTT) y los Tecnológicos Superiores Descentralizados (TESD) recae en un grupo de personas compuesto por representantes del gobierno federal y estatal, así como de representantes de empresarios de la localidad. Sin embargo, en las UUPP, además de estos actores, también participa el BID a través de los lineamientos establecidos en el Proforhcom. El programa es implementado por la Secretaría de Educación Pública (SEP) a través de la UCAP y del Codipro.

Pareciera que el BID tiene una gran influencia en las funciones y atribuciones de las UUPP. Mientras que todas las IEST del país cuentan con un reglamento interno regulado por la SEP, las atribuciones y funciones de la CUP se encuentran reguladas por el reglamento operativo del Proforhcom, aprobado por el Codipro y, con el visto bueno del BID.

Muchos de los principios de la NGP se encuentran representados en las prácticas laborales del personal de la CUP. La gran mayoría del personal de la CUP son consultores y 
trabajan bajo el régimen de honorarios. Si bien, a través de esta medida se logran incrementar los ingresos públicos, la satisfacción de los diferentes actores es relativa. En el caso del personal de la CUP, muchos de ellos se quejan de que el pago por honorarios significa no contar con una estabilidad laboral, seguridad social o prestaciones sociales.

Asimismo, la política a posteriori se pudo observar en las propuestas que realizaba el gobierno a través de la SEP ante el BID. En la "propuesta" del Proforhcom Fase I, se acordó lo que ya se había realizado hasta ese momento con las UUPP, como fueron el índice de deserción, así como determinadas carreras y, capacitación de profesores con el MEBCP. No obstante, también se llegó a acordar ciertas metas a futuro, como fue el caso de que las UUPP serían las encargadas de coordinar y dirigir a las Universidades Tecnológicas (UUTT) para implementar el MEBCP.

Aunque todavía no había concluido el Proforhcom Fase I, en Diciembre del 2008 se dio a conocer la propuesta de préstamo para el Proforhcom Fase II. El Proforhcom Fase I, terminó en abril del 2010 y en febrero del 2010 se estableció el contrato para iniciar el Proforhcom Fase II (BID, 2009, 2010).

Se podría decir que las evaluaciones que realiza el BID son apresuradas y, responden más a una lógica de compromiso que a los mismos resultados de los programas. Este hecho se pudo mostrar cuando el mismo BID declaró que las metas del Proforhcom Fase I se habían cumplido, cuando aún le faltaban dos años para terminar dicho programa. Así en el 2008, el BID decidió establecer nuevos compromisos a través del Proforhcom Fase II.

\section{Referencias}

BANCO INTERAMERICANO DE DESARROLLO - BID. Programa de Formación de recursos humanos basada en competencias Proforhcom - Fase I (ME-0250). Propuesta de Préstamo. Mexico, 2004.

Contrato de préstamo No. 1579/OC-ME entre los Estados Unidos Mexicanos y el Banco Interamericano de Desarrollo. Programa de Formación de Recursos Humanos Basada en Competencias, Fase I. Mexico, 2005.

. Programa de formación de recursos humanos basada en competencias

Proforhcom - Fase II (Me-L1039). Perfil de Proyecto. Mexico, 2008.

. Programa de formación de recursos humanos basada en competencias

Proforhcom - Fase II (Me-L1039). Propuesta de Préstamo. Mexico, 2009. 
Contrato de Préstamo No. 2167/OC-ME entre los Estados Unidos Mexicanos y el Banco Interamericano de Desarrollo. Programa de Formación de Recursos Humanos Basada en Competencias, Fase II. Mexico, 2010.

BRAUN, D.; MERRIEN, F. Towards a new model of governance for universities. London: Jessica Kingsley Publisher, 1999.

INTER-AMERICAN DEVELOPMENT BANK - IDB. Disponible en: <www.iadb.org>. Acseso en: 17 mayo 2011.

KENT, R. K.; DIDOU, S.; VRIES, W. Reformas financieras en las universidades públicas en México: los años noventa. In: KENT SERNA, R. et al. Experiencias de reforma en la educación superior en América Latina: los años noventa. Madrid: Plaza y Valdez, 2001. p. 229-275.

LYNN, L. Reforma a la gestión pública: tendencias y perspectivas. In: PARDO, M. C. De la administración pública a la gobernanza. México: Colmex, 2004. p. 105-137.

MAGALHAES, A.; AMARAL, A. Mapping out discourses on higher education governance. In: HUISMAN, J. International perspective on the governance of higher education. Nueva York: Routledge, 2009. p. 182-197.

MEXICO. Subsecretaría de Educación Superior. Coordinación General de Universidades Tecnológicas y Politécnicas. Disponible en: <http://politecnicas.sep.gob.mx>. Acceso en: 13 mar. 2007.

Subsecretaría de Educación Superior. Disponible en: <www.ses.sep.gob.mx>. Acseso en: 30 mayo 2011.

NEAVE, G. On the cultivation of quality, efficiency and enterprise. An overview of recent trends in higher education in Western Europe 1986-1988. European Journal of Education, Oxford, v. 25, n. 2-3, p. 273-283, 1988.

NEAVE, G.; Van VUGHT, F. Government and Higher education relationships across three continents: the winds of change. Oxford: Pergamon, 1994. (Series: Issues in Higher Education).

UNIDAD COORDINADORA Y ADMINISTRADORA DEL PMETYC - UCAP. Síntesis del PMETYC I 1995-2003 y Estructura del Proforhcom. México, 2007. Área de Promoción y Asistencia Técnica, Pmetyc, Documento Interno.

Recebido em: 30/11/2014

Aprovado em: 19/04/2015 